\title{
A renal oncocytoma in a Maltese dog with renal cysts
}

\author{
Jong-uk Kim ${ }^{1}$, Jinsung Park ${ }^{1}$, Gonhyung Kim ${ }^{2}$, Dongwoo Chang ${ }^{3}$, Byeongwoo Ahn ${ }^{1 *}$ \\ ${ }^{1}$ Laboratory of Veterinary Pathology, \\ ${ }^{2}$ Laboratory of Veterinary Surgery, and \\ ${ }^{3}$ Laboratory of Veterinary Medical Imaging, Veterinary Medical Center and College of Veterinary Medicine, \\ Chungbuk National University, 1 Chungdae-ro, Seowon-gu, Cheongju 28644, Korea
}

\begin{abstract}
A renal oncocytoma was diagnosed in an 8-year-old female Maltese dog with a history of renal cysts. Tumor cells were not detected until six months after observation of renal cysts. Nephrectomy was performed to treat the neoplasia. Tumor-like masses with numerous nodules were observed in the inner surface of cysts present in the caudal part of the left kidney. Histologically, the tumor consisted of cells with abundant eosinophilic cytoplasm. The diagnosis was based on histological features, periodic acid-Schiff reaction, and immunohistochemical cytokeratin staining. Based on a literature review, this is the first canine renal oncocytoma case reported in Korea.
\end{abstract}

Keywords: canine, renal oncocytoma, renal cysts

\section{${ }^{*}$ Corresponding author}

Byeongwoo Ahn

Laboratory of Veterinary Pathology, Veterinary Medical Center and College of Veterinary Medicine, Chungbuk National University, Cheongju 28644, Korea.

Tel: $+82-43-271-3246$

Fax: +82-43-261-2508

E-mail: bwahn@cbu.ac.kr

ORCID:

Gonhyung Kim

https://orcid.org/0000-0002-9384-4741

Dongwoo Chang

https://orcid.org/0000-0002-7721-773X

Byeongwoo Ahn

https://orcid.org/0000-0002-4112-7724

Conflict of Interest

The authors declare no conflicts of interest.

Received: November 19, 2018

Revised: January 26, 2019

Accepted: February 11, 2019
Canine renal oncocytoma is an extraordinarily rare, usually benign, tumor composed of cells with brightly eosinophilic cytoplasm. In humans, the occurrence of oncocytoma is relatively common. It has been reported in a variety of organs including salivary gland, endocrine glands, liver, lungs, and kidneys in humans [1]. Oncocytoma has been sporadically reported in cats, dogs, and rats [2-5]. The first report of a canine renal oncocytoma was documented in $2000[2,3]$. Histological features include eosinophilic granular cytoplasm with periodic acid-Schiff (PAS) positive granules and an abundance of mitochondria [6]. Immunohistochemistry (IHC) for cytokeratin and vimentin has been utilized for the diagnosis of this tumor as it expresses cytokeratin AE1/AE3, but not vimentin [7, 8]. In this study, we report a case of canine renal oncocytoma.

An 8-year-old female Maltese dog was referred to the Veterinary Medical Center of Chungbuk National University due to abdominal distension. On physical examination, a soft large mass was palpated in the abdomen. By ultrasonography, a round to oval cystic lesion containing fluid-attenuation material was observed in the caudal part of the left kidney. Radiography revealed a mass $\left(6.5 \times 5.3 \times 6.4 \mathrm{~cm}^{3}\right)$ with round-shaped soft tissue opacity (Fig. 1A). Aspirations were performed to treat cystic swelling caused by continuous fluid secretion. The mass was not surgically removed until 6 months later when tumor cells were detected by cytological examination of the fluid.

Grossly, there were clear fluid and tumor-like masses in the swollen cystic space present in the caudal part of the kidney. The diameter of the long axis of the tumor was $6 \mathrm{~cm}$. The tumor tissues consisted of relatively large white masses and a number of tan-colored small nodules scattered throughout the cysts (Fig. 1B).

Tissues samples were fixed in 10\% phosphate-buffered formalin, processed and embedded in paraffin for histopathological examinations. Four-micrometer-thick tissue sections were used for staining with hematoxylin and eosin, PAS, and IHC for cytokeratin AE1/AE3 and vimentin. For IHC, monoclonal anti-pancytokeratin AE1/AE3 (1:1, Abcam, UK) and monoclonal anti-vimentin $(1: 400$, Abcam) were used as primary antibodies. Binding of primary antibodies was detected by applying an avidin-biotin-peroxidase complex method (ABC kit; Vector Laboratories, USA). 

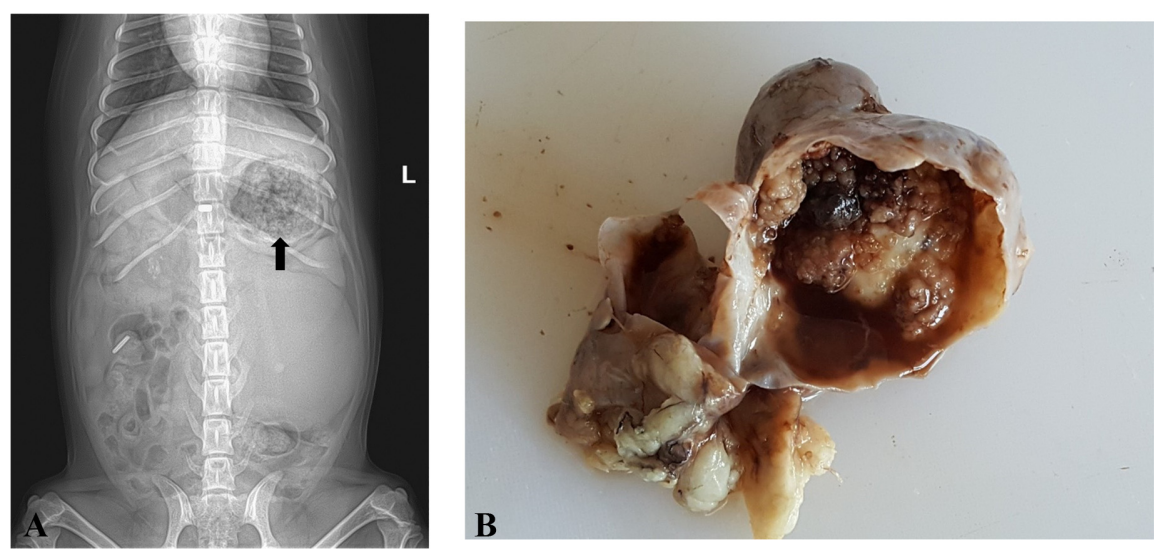

Fig. 1. Ventrodorsal radiograph of the abdomen showing a round-shaped mass (arrow) with a smooth margin on the left side (A). Note the numerous tan or white tumor masses and watery fluid in the cyst fixed in formalin (B).
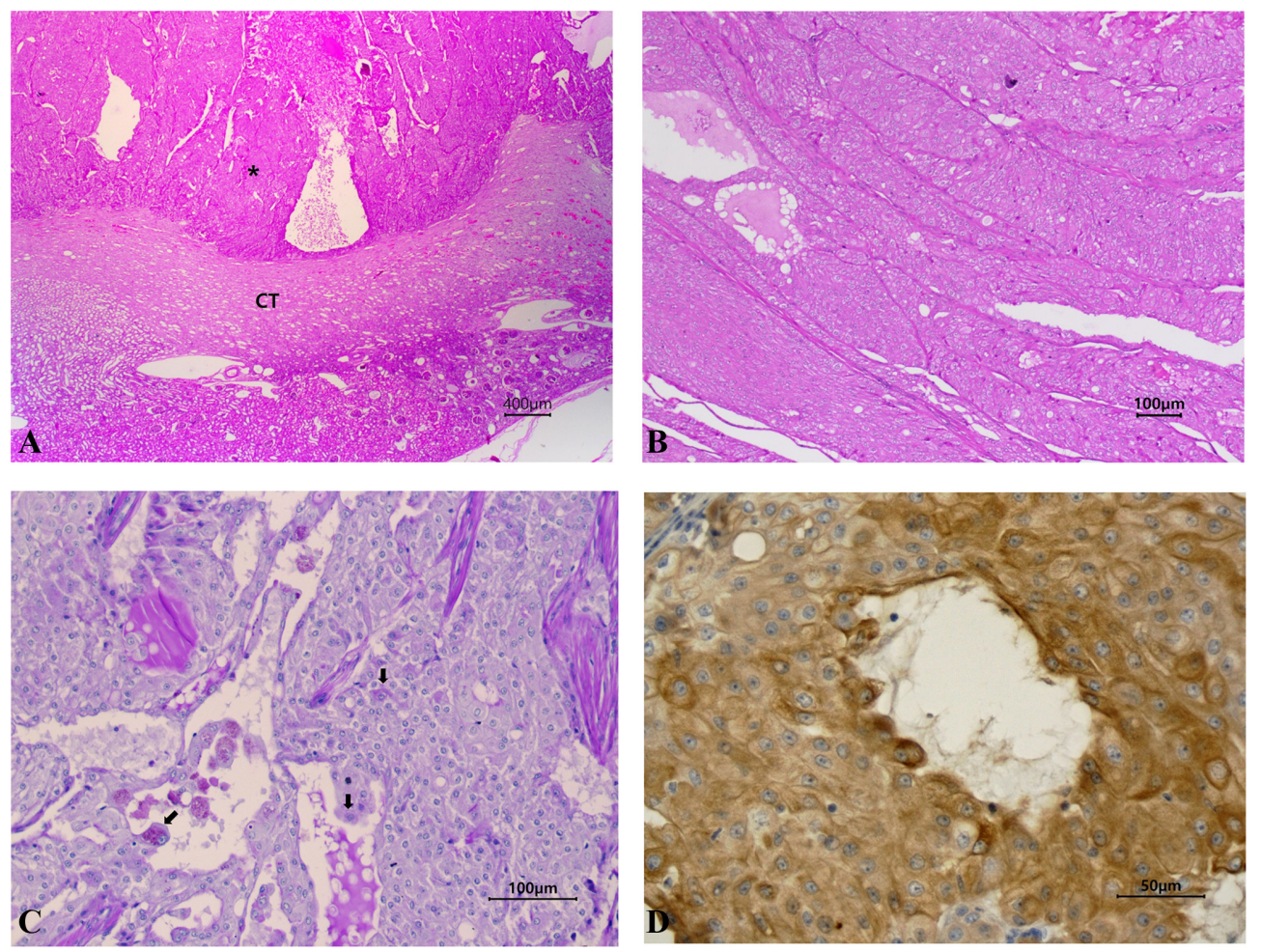

Fig. 2. Histological features. Note that the tumor tissue (asterisk) surrounded by thick connective tissues (CT) compresses the renal cortex $(\mathrm{A}, \times 25)$. The large eosinophilic tumor cells in lobular architectures were arranged in solid or tubular patterns. Homogeneous eosinophilic fluid was observed in lumens $(\mathrm{B}, \times 100)$. Tumor cells show positive reactions for PAS staining $($ arrows $)(C, \times 200)$ and cytokeratin AE1/AE3 (D, avidin-biotin complex method, $\times 400)$.

Histologically, the mass was well demarcated from the parenchyma by fibrous stromal tissues. There were no normal renal histological structures such as glomerulus or renal tubules in these cysts (Fig. 2A). In some area, cortical tissues were compressed by the tumor. Neoplastic cells were packed into lobular architectures by well-developed thin fibrous stromal tissues. Some tumor cells in small nodules showed papillary growth. Tumor cells in lobular architectures were arranged in solid or tubular patterns. Contained cellular debris or homogeneous eosinophilic fluid was observed in lumens. Tumor cells were well differentiated, round to oval to polygonal in shape, and large with intensely eosinophilic granular cytoplasm and distinct cell borders. Nuclei were round with coarsely stippled chromatin and a prominent nucleolus (Fig. 2B). Cytoplasmic fine granules were moderately positive in PAS staining (Fig. 2C). IHC revealed that cytokeratin AE1/AE3 was expressed in the cytoplasm of tumor cells (Fig. 2D); however, vimentin was not expressed 
at all (data not shown).

In the present case, there were some histologic features of benign tumors, including expansive growths, well-differentiated and uniform cellular appearances, non-metastasis, and few mitotic figures. The diagnostic criteria of oncocytoma include distinctive granular eosinophilic cytoplasm, positive PAS reaction, immunoexpression of cytoplasmic cytokeratin, but not vimentin, abundant mitochondria, and absence of ultrastructural vesicles $[6,9]$. Presence of an eosinophilic variant of chromophobe renal cell carcinoma (RCC) is considered a differential diagnosis of renal oncocytoma. Although histological features of oncocytoma are quite similar to those of chromophobe RCC, chromophobe RCC is known to be negative in PAS staining but positive in colloidal iron staining. In addition, oncocytoma has ultrastructural vesicles. Immunohistochemical expression of some markers is useful when differentiating the diagnosis of $\mathrm{RO}$ and chromophobe RCC from RCC. While RCC expresses both cytokeratin and vimentin, RO and chromophobe RCC express cytokeratin only [2]. Based on the diagnostic criteria of oncocytoma and chromophobe RCC, this case was diagnosed to be canine renal oncocytoma. The histogenesis of oncocytoma is unclear. However, tumor cells are thought to be derived from intercalated cells of collecting ducts in humans $[9,10]$. The cysts containing tumor masses and fluid in this case may be identical to classic renal cysts. Although the pathogenesis of renal cysts has not been fully elucidated, these cysts are likely to be derived from normal or noncystic segments of the nephron. Therefore, renal cysts normally occur at renal tubules, collecting ducts and/or Bowman's space [11]. Lumenal surfaces of these cysts are lined by flattened epithelium. In this case, there were numerous small cysts lined by epithelial cells in the non-tumorous parenchymal cortex, indicating that this case might have some genetic factors involved in the genesis of the cysts. Being separated from the parenchyma and not lined by epithelium because of tumor cell proliferation on the thick connective tissues, tumor-bearing large cysts are considered to be the same cyst type as those present in the normal cortex. However, the causative relationship between renal cysts and neoplasia is undescribed. Based on our literature review, this is the first case of a canine oncocytoma reported in Korea.

\section{Acknowledgements}

This work was supported by the research fund of Chungbuk National University in 2016.

\section{References}

1. Ghadially FN. Ultrastructural Pathology of the Cell and Matrix. 3rd ed. Butterworths, London, 1988.

2. Buergelt $\mathrm{CD}$, Adjiri-Awere A. Bilateral renal oncocytoma in a Greyhound dog. Vet Pathol 2000;37:188-192.

3. Dempster AG, Delahunt B, Malthus AW, Wakefield JS. The histology and growth kinetics of canine renal oncocytoma. J Comp Pathol 2000;123:294-298.

4. Kanjo M, Mitsumori K, Maita K, Shirasu Y. Pinnal oncocytoma in a rat. Vet Pathol 1990;27:292-294.

5. Lee S, Choi HJ, Lee HB, Jo SM, Mun JH, Son WC. Renal oncocytoma in a cat with chronic renal failure. JFMS Open Rep 2017;3:2055116917693491.

6. Meuten DJ. Tumors in domestic animals. 5th ed. Wiley Blackwell, Ames, 2017.

7. Beham A, Ratschek M, Zatloukal K, Schmid C, Denk H. Distribution of cytokeratins, vimentin and desmoplakins in normal renal tissue, renal cell carcinomas and oncocytoma as revealed by immunofluorescence microscopy. Virchows Arch A Pathol Anat Histopathol 1992;421:209-215.

8. Bonsib SM, Bromley C, Lager DJ. Renal oncocytoma: diagnostic utility of cytokeratin-containing globular filamentous bodies. Mod Pathol 1991;4:16-23.

9. Eble JN, Young RH. Tumors of the urinary tract. In: Fletcher C (ed.). Diagnostic Histopathology of Tumors. 2nd ed. pp. 475-565, Churchill Livingstone, New York, 2000.

10. Cohen C, McCue PA, Derose PB. Histogenesis of renal cell carcinoma and renal oncocytoma. An immunohistochemical study. Cancer 1988;62:1946-1951.

11. Zachary JF, McGavin MD. The Pathologic Basis of Veterinary Disease. 5th ed. Elsevier, St. Louis, 2012. 